\title{
A protein-based phylogenetic tree for Gram-positive bacteria derived from hrcA, a unique heat-shock regulatory gene
}

\author{
Suhail Ahmad, ${ }^{1,2}$ Angamuthu Selvapandiyan ${ }^{1}$ and Raj K. Bhatnagar \\ Author for correspondence: Raj K. Bhatnagar. Tel: +9111618 1242. Fax: +91116162316. \\ e-mail: rajbhatnagar@hotmail.com
}

1 International Centre for Genetic Engineering and Biotechnology, Aruna A. Ali Marg, PO Box 10504, New Delhi 110067, India

2 Department of Microbiology, Faculty of Medicine, Kuwait University, PO Box 24923, Safat 13110, Kuwait

\begin{abstract}
The dnaK operon from Bacillus subtilis and other Gram-positive bacteria with low G+C DNA content contains additional heat-shock genes, including hrcA. The hrcA gene encodes a transcription factor that negatively regulates heatshock genes and is uniformly present in all Gram-positive bacteria studied to date. An hrcA homologue is also present in Synechocystis species, Leptospira interrogans, Chlamydia trachomatis, Caulobacter crescentus and Methanococcus jannaschii, organisms that diverged early on from the common ancestor of all Gram-positive bacteria and Proteobacteria, according to 165 rRNA phylogeny. A partial, protein-based phylogenetic tree, derived using amino acid sequence homology of hrcA proteins from Gram-positive bacteria, is presented here, and the results are compared with the phylogenetic trees generated from 165 rRNA, dnaK and dnaJ sequences. The location of the hrcA gene and the genome organization of the dnaK operon support the division of all Gram-positive bacteria into three major groups: one group contains highG+C Gram-positive bacteria, and two others contain low-G+C Gram-positive bacteria. Among the Gram-positive bacteria with low G+C DNA content, the results indicate that there is a close phylogenetic relationship between Bacillus species and Clostridium species on the one hand and between Lactococcus lactis and Streptococcus mutans on the other. Streptomyces and Mycobacterium species also exhibited a close relationship. A hierarchical arrangement of Gram-positive bacteria based on HrcA sequences is proposed as an additional refinement of the phylogenetic relationships within this important bacterial group.
\end{abstract}

Keywords: $h r c A$, dnaK operon, Gram-positive bacteria, phylogeny

\section{INTRODUCTION}

The evolutionary relationships among microorganisms are being determined by partial or complete sequencing of $5 \mathrm{~S}$ and 16S rRNA genes (Olsen \& Woese, 1993; Stackebrandt \& Goebel, 1994; Stackebrandt et al., 1997; Woese, 1994) mainly because their resistance to evolutionary change allows the entire phylogenetic span of ancient and modern prokaryotes to be analysed simultaneously. However, it has been shown that the resolution power of rRNA sequences is limited when closely related organisms that diverged at almost the same time are being

Abbreviation: HSP, heat-shock protein. examined (Fox et al., 1992; Woese, 1987). More recently, sequencing data for other highly conserved molecules (e.g. heat-shock proteins, HSPs) have been used because of their ubiquity and high degree of sequence conservation. Thus, phylogenetic analyses based on Hsp40 (DnaJ), Hsp60 (GroEL), Hsp70 (DnaK) and Hsp90 family of protein sequences have been carried out recently (Boorstein et al., 1994; Bustard \& Gupta, 1997; Falah \& Gupta, 1994; Gupta, 1995; Gupta \& Golding, 1993; Gupta et al., 1989). These analyses have revealed a number of important differences relative to rRNA-based phylogenies (Boorstein et al., 1994; Brown et al., 1994; Gupta \& Golding, 1993). However, highly conserved molecules (e.g. 16S rRNA, Hsp70) may not be suitable for determining evolutionary relationships among bac- 
(a) $16 \mathrm{~S}$ rRNA sequences

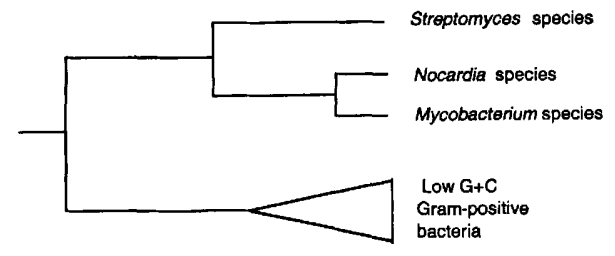

(b) Hsp70 (DnaK) sequences

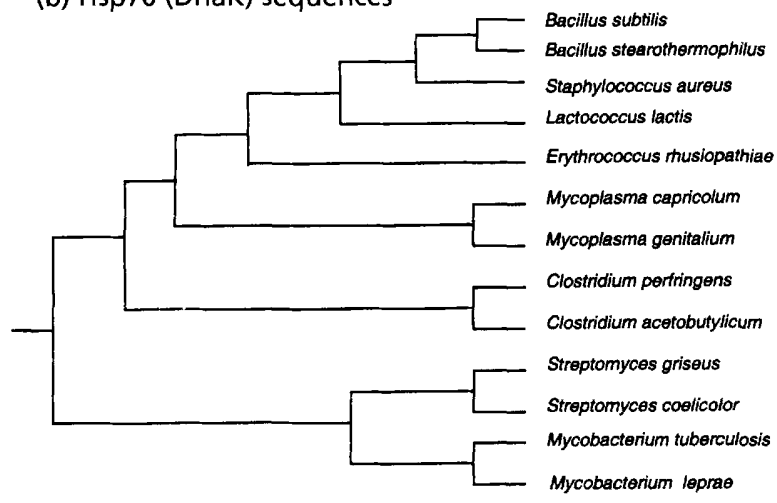

(c) Hsp40 (DnaJ) sequences

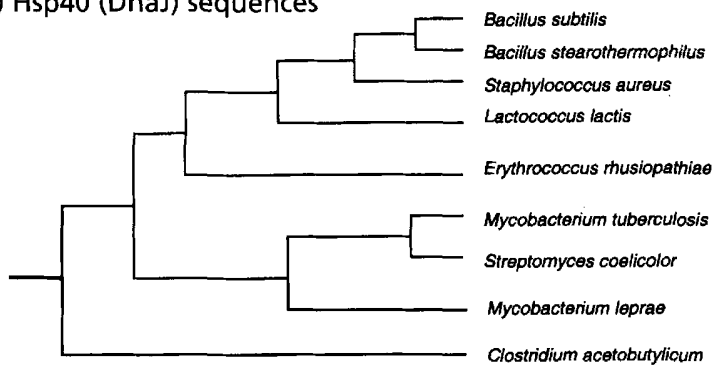

Fig. 1. Comparison of phylogenetic relationships among Grampositive bacteria deduced from (a) 165 rRNA sequences (Olsen \& Woese, 1993; Stackebrandt et al., 1997; Woese, 1987), (b) the amino acid sequence homology of DnaK proteins (Falah \& Gupta, 1997; Gupta \& Golding, 1993) and (c) DnaJ protein sequences (Bustard \& Gupta, 1997). The trees show the branching order rather than the actual distances. (a) The genera representing Gram-positive bacteria with low-G + C DNA content include, among others, Bacillus species, Clostridium species, Staphylococcus species, Lactococcus species, Streptococcus species and Mycoplasma species.

terial groups that diverged at almost the same time; amino acid sequence homologies, for other proteins that are not so highly conserved, and gene-fusion events may be more appropriate methods (Ahmad \& Jensen, 1989; Jensen \& A hmad, 1990). Furthermore, a study based on a very small number of genes could give misleading results if one of the genes had been horizontally transferred across bacterial groups at an earlier stage of divergence. Thus, greater resolution of evolutionary branching can be achieved only by using a greater number of evolutionary trees.

The Gram-positive group of bacteria is of special microbiological interest because of pathogenic and non-pathogenic relationships with mammalian systems, as well as because of its potential for industrial exploitation. The Gram-positive bacteria have evolved only recently from a common ancestor (Woese, 1987). Their recent evolutionary origin means that highly conserved molecules, such as $16 \mathrm{~S}$ rRNA sequences, could not resolve the branching order, as the divergence among various groups occurred at almost the same time (Rainey et al., 1995; Stackebrandt et al., 1997; Woese, 1987). Thus, comparative sequence analyses of other cistrons that are less highly conserved may help to clarify the hierarchical order.

A comparison of the phylogenetic trees constructed by means of 16S rRNA sequencing (Stackebrandt et al., 1997; Woese, 1987) and amino acid sequence homology of DnaK (Falah \& Gupta, 1994; Gupta \& Golding, 1993) and DnaJ (Bustard \& Gupta, 1997) proteins for several Gram-positive bacteria is shown in Fig. 1. Almost the same set of organisms are used. The resolution and the discrepancies in the branching order in the three trees are clear. The order of branching among Gram-positive bacteria with low G +C DNA content could not be resolved on the basis of $16 \mathrm{~S}$ rRNA sequences. The tree based on Hsp70 sequences depicts Clostridium species branching off early before the divergence of Mycoplasma/Lactococcus/ Staphylococcus species from the Bacillus species. Both Bacillus species and Clostridium species sporulate (Errington, 1993), a process that involves the expression of several genes. The tree based on DnaJ sequences shows Clostridium species branching off from the low-G +C DNA organisms (e.g. Bacillus species) even earlier than other high-G +C DNA organisms (Bustard \& Gupta, 1997). Furthermore, the branching order places Mycobacterium tuberculosis closer to Streptomyces coelicolor than to Mycobacterium leprae, a result that is highly unlikely to be valid (Cole et al., 1998).

The discrepancies in the branching order could be because of horizontal transfer of a gene at an earlier point of divergence or because of different mutation rates for these genes in various organisms following their divergence. The genes encoding HSPs are ubiquitous and essential for cell survival. However, the possibility that horizontal gene transfer is responsible for the varying results obtained with DnaK and DnaJ phylogenies cannot be ruled out in view of the presence of more than one gene copy for dnaJ homologues in some Gram-positive bacteria (Grandvalet et al., 1998). Both of the above points can be effectively addressed and the branching order resolved by simply analysing a greater number of carefully chosen cistrons; this is the basis of the study presented here.

The dnaK operon from Bacillus subtilis, Bacillus sphaericus and other Gram-positive bacteria contains two, three or several other heat-shock genes, including hrcA, grpE and dnaJ (Ahmad et al., 1999; Eaton et al., 1993; Falah \& Gupta, 1997; Mogk \& Schumann, 1997; Narberhaus et al., 1992; Ohta et al., 1994; Tokunaga et al., 1998; Wetzstein et al., 1992). The $h r c A$ gene encodes a novel, heat-shock regulatory gene 
(Roberts et al., 1996; Schulz \& Schumann, 1996) that is distributed throughout Gram-positive bacteria and is an ideal basis for constructing phylogenetic relationships. In this paper, we present a phylogenetic tree for several Gram-positive bacteria, based on the genome location of the $h r c A$ gene (relative to the $d n a K$ operon) and, for the first time, amino acid sequence homology comparisons for the HrcA protein.

\section{METHODS}

The cloning, sequencing and genome organization of the dnaK operon from $B$. sphaericus has been described previously (unpublished; EMBL accession no. Y17157). The dnaK operon contains at least five protein-coding genes in the order $h r c A-g r p E-d n a K-d n a J-O R F 35$ (Ahmad et al., 1999). The $h r c A$ gene was translated and a FASTA search of EMBL, GenBank and other databases was performed using the encoded protein sequence (Altschul et al., 1990). The sequences of $h r c A$ homologues from various Gram-positive bacteria, identified in FASTA searches, were retrieved. The EMBL accession numbers of the hrc $A$ homologous sequences from the database are as follows: B. subtilis, P25499; Bacillus stearothermophilus, O45550; Clostridium acetobutylicum, P30727; Staphylococcus aureus, P45556; Lactococcus lactis, P42370; Streptococcus mutans, O06940; Mycoplasma pneumoniae, P75351; Mycoplasma genitalium, P47447; Mycoplasma capricolum, U51235; Mycobacterium tuberculosis, O05824; Mycobacterium leprae, Q49772; Streptomyces albus, AF025656; Leptospira interrogans, G2735759; Synechocystis species, P72795; Chlamydia trachomatis, P54306; and Caulobacter crescentus, U33324. Various $h r c A$-encoded sequences were initially aligned using the CLUSTAL program (PC/GENE software) (Myers \& Miller, 1988 ) and then inspected for any obvious misalignments. The amino acid sequence identity between pairs of proteins was calculated for the entire length of all HrcA proteins by using the FASTA program (Altschul et al., 1990). The partial sequence from Mycobacterium leprae was not used for pairwise comparisons. However, the phylogenetic analyses were carried out with the $195 \mathrm{~N}$-terminal amino acid residues of all $h r c A$ homologues corresponding to the partial sequence of HrcA protein that is available for the $M y c o-$ bacterium leprae homologue. A phylogenetic dendrogram was constructed using the treeing algorithms contained in the PHYLIP (version 3.5) phylogenetic software package. The robustness of the tree topologies was evaluated by bootstrap analysis (100 resamplings) of the neighbour-joining data.

\section{RESULTS AND DISCUSSION}

The dnaK operon from $B$. sphaericus encodes at least five heat-shock proteins arranged in the order $h r c A-$ grpE-dnaK-dnaJ-ORF35. The location and genome organization of the $h r c A$ gene in relation to the other $d n a K$-related genes ( $h r c A, \operatorname{grp} E, d n a K$ and $d n a J)$ of the dnaK operon were compared using various Grampositive bacteria and other bacterial groups that possess an $h r c A$ homologue. The results are shown in Fig. 2. The organization of these four heat-shock genes is identical in one cluster of Gram-positive bacteria that have low $\mathrm{G}+\mathrm{C}$ content in their DNA; that cluster included Bacillus species, Clostridium species, Staphylococcus species and Mycoplasma species (Ahmad et al., 1999; Falah \& Gupta, 1997; Mogk \& Schumann, 1997; Narberhaus et al., 1992; Ohta et al., 1994; Tokunaga et al., 1998; Wetzstein et al., 1992). A similar gene arrangement within the dnaK operon is also found in the spirochaete Leptospira interrogans (Ballard et al., 1998). Although the hrcA gene is part of the dnaK operon in both Lactococcus lactis and Streptococcus mutans, the dnaJ gene is not encoded by the dnaK operon (Eaton et al., 1993; Jayaraman et al., 1997; Koch et al., 1998). The gene order hrcAgrpE-dnaK found within the dnaK operon of both Lactococcus lactis and Streptococcus mutans is unique to these Gram-positive bacteria with low G + C DNA content. The chromosome location of the $h r c A$ gene and the organization of the $d n a K$ operon, however, differ in Gram-positive bacteria with high $\mathrm{G}+\mathrm{C}$ DNA content. The hrc $A$ gene is present independently, while the $d n a K$ operon contains the remaining three genes. Furthermore, the gene order dnaK-grpE-dnaJ within the dnaK operon is unique to the high-G + C DNA group of Gram-positive bacteria examined to date (Bucca et al., 1995; Cole et al., 1998; Grandvalet et al., 1998).

Thus, the chromosome location and genome organization of the four heat-shock genes ( $h r c A$, grpE, $d n a K$ and $d n a J$ ) allow the categorization of all Grampositive bacteria into three groups. One group includes high-G $+\mathrm{C}$ Gram-positives, whereas low-G +C Gram-positives fall into two groups. The clusters roughly reflect the grouping of these organisms on the basis of 16S rRNA sequencing data (Olsen \& Woese, 1993; Stackebrandt et al., 1997; Woese, 1987).

The $h r c A$-encoded protein sequence from $B$. sphaericus was compared with all the protein sequences in various databases using FASTA searches and the homologous sequences were retrieved. Thus, a total of 13 complete HrcA protein sequences, in addition to a partial sequence for Mycobacterium leprae, were obtained from various Gram-positive bacteria. These sequences corresponded to both of the major groups (high $\mathrm{G}+\mathrm{C}$ DNA content and low G + C DNA content) of Grampositive bacteria. A multiple alignment based on $h r c A$ encoded amino acid sequences from Gram-positive bacteria is shown in Fig. 3. Two highly conserved regions have been identified from all the $h r c A$ sequences (labelled in Fig. 3 as Box 1 and Box 2). The $\mathrm{N}$-terminal 80 amino acids of the $h r c A$ protein sequences exhibit maximum identity and contain one of the two highly conserved domains (Fig. 3, Box 1). This is similar to the amino acid sequence identities observed with Hsp40 (DnaJ) sequences (Bustard \& Gupta, 1997).

The FASTA program and the PALIGN program (PC/GENE software) were used to make a pairwise comparison of the $h r c A$-encoded protein sequences from representative species of Gram-positive bacteria. The amino acid sequence of the HrcA protein from Synechocystis species (Glatz et al., 1997) was included because of the reportedly close association between cyanobacteria 


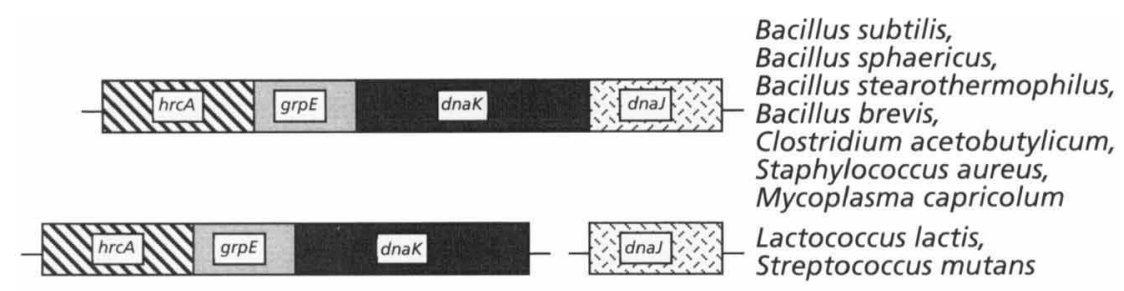

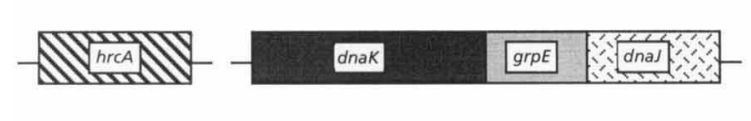

Mycobacterium tuberculosis, Mycobacterium leprae, Streptomyces coelicolor, Streptomyces albus
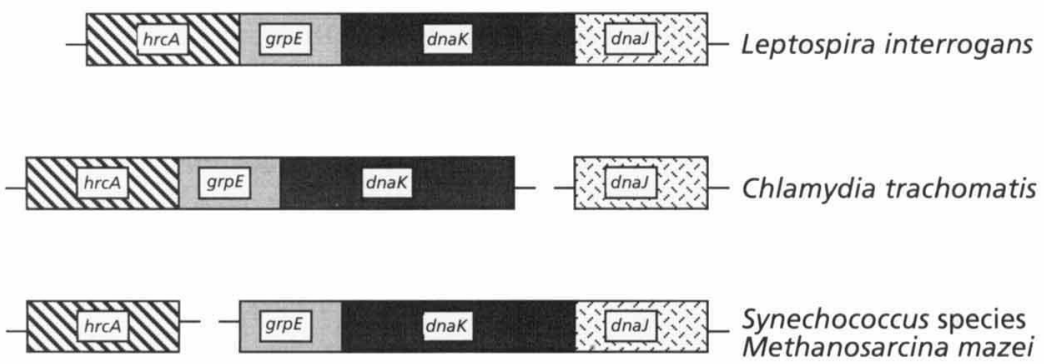

dnak Escherichia coli
Fig. 2. Chromosomal locations of hrcA gene homologues and the organization of the dnak operon from diverse Gram-positive bacteria, including Leptospira interrogans (Spirochaetales) (Ballard et al., 1998), Chlamydia trachomatis (Chlamydiales) (Tan et al., 1996), Synechococcus species (cyanobacteria), Methanosarcina mazei (Archaea) (Conway de Macario et al., 1994) and Escherichia coli (Proteobacteria) (Lipinska et al., 1988; Saito \& Uchida, 1978). The chromosome locations of hrcA homologues are shown in relation to the other genes encoded by the dnaK operon. Although Leptospira interrogans and C. trachomatis have gene arrangements similar to those exhibited by the first and second groups of low-G +C DNA, Gram-positive bacteria, they are shown separately because the other genes in the vicinity vary. and Gram-positive bacteria (Bustard \& Gupta, 1997; Woese, 1994). This analysis indicated that the amino acid identity between various homologues varied between 24 and $80 \%$ over the entire length of these proteins (Table 1). The hrcA homologues from various Bacillus species exhibited maximum identity to each other and significantly higher homology with the corresponding protein from Clostridium acetobutylicum, another spore-forming, Gram-positive bacterium with low $\mathrm{G}+\mathrm{C}$ DNA content relative to $\mathrm{HrcA}$ sequences from other Gram-positive bacteria (Table 1). The amino acid sequence of the $h r c A$ homologue from Mycobacterium leprae is known only for the Nterminal 195 residues and exhibited $90 \%$ identity with the corresponding protein from Mycobacterium $t u$ berculosis (data not shown). The two mycobacterial sequences also exhibited higher amino acid identity with the corresponding protein from Streptomyces albus (another Gram-positive bacterium with high $\mathrm{G}+\mathrm{C}$ DNA content) than they did with other sequences. For a large number of sequence pairs, the amino acid identity was in the range $24-32 \%$, which is similar to the overall identity observed for DnaJ protein sequences (Bustard \& Gupta, 1997) but lower than that observed for Hsp70 protein sequences (Falah \& Gupta, 1994). This suggests that HrcA and DnaJ proteins are structurally not as highly conserved as the Hsp 70 protein.

The amino acid sequence alignment of $h r c A$ homologues shown in Fig. 3 was used to examine the phylogenetic relationships between various Grampositive bacteria. For this purpose, only the sequence of the $195 \mathrm{~N}$-terminal amino acid residues from various $h r c A$ homologues was utilized, including the partial sequence from Mycobacterium leprae. The phylogenetic relationships between various Gram-positive bacteria were deduced by using the PHYLIP phylogenetic software package. To determine the reliability of the branching order, the sequences were bootstrapped 100 times (Felsenstein, 1985).

A neighbour-joining consensus tree based on HrcA sequences is shown in Fig. 4. The bootstrap scores observed for all the nodes are indicated. In general, the long branch lengths at deeper positions are characterized by low bootstrap scores. No members belonging to the low-G $+\mathrm{C}$ DNA group of Grampositive bacteria share an immediate common ancestor. The three Bacillus species, Clostridium acetobutylicum, and Staphylococcus aureus are clustered together. Clostridium acetobutylicum branched off before the branching of the common ancestor of the Bacillus species, while Staphylococcus aureus branched off at the deepest position within this cluster. Other members belonging to the low-G $+\mathrm{C}$ DNA content category failed to cluster within this grouping. Lactococcus lactis exhibited a close relationship with Streptococcus mutans. However, these bacteria, together with Mycoplasma species belonging to the low-G + C DNA group, diverged before the Grampositive bacteria with high $\mathrm{G}+\mathrm{C}$ DNA content, i.e. from the main lineage of low $\mathrm{G}+\mathrm{C}$ group. These clusters are characterized by long branch lengths, perhaps indicating a tentative hierarchical order at deeper positions. It is interesting to note that 


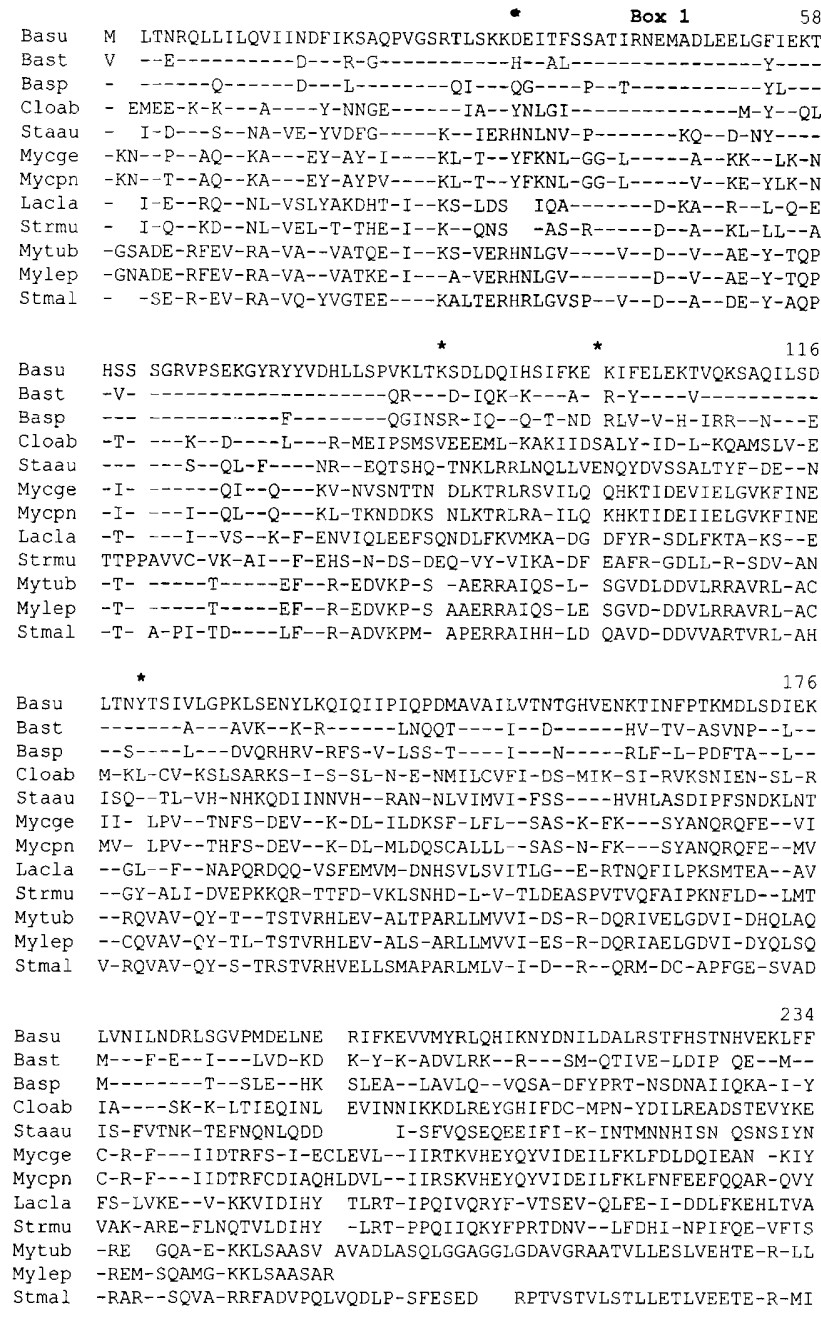

293

Basu GGKINMLNQPEFHDITRVRSLLSIEKEQDVLKLVQSP HT GISIKIGKENDYEEMENC

Bast A--T---1--N--QKI-P-MKM--Q-K-FYR-LRKHNRK --QVT--R--QLS---

Bast A--T-----N--QKI-P-MKM--Q-K-FYR-LRKHNRK --QVT--R--QLS---

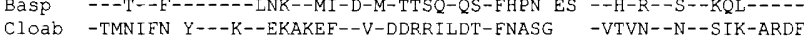

Staau ---VKLIDALNESNVSSIQPT-QY--SNRIAEL-QDISSEN -NV-----I-DS LSDI

MYCge -IQYLAK ----ANQEKLTKI-N-L-DTSVWQQMAFINQTNQKTN-VF-DQLGFK EI

MycPn -IHYI.AQ ----ANQERLTRI-N-L-DTSVWQQMAFMNQNNQTTN-TF-DKLGL-GE EV

Lacla -HKNIFDYATDNL AELYK-F-DD-RMLHEIREITNN DE MRAVKFDND EKFMKNL

Strmu -KIKTLEFAG LDTYQF-ENLQSVALEIRQSLPE DE LHRVQVADSKEKSLADI

Mytub --TA-LT RNAADFGGSL--I-EAL--QVV--R-LAAQQEAGKVTVR--H-TAS-Q-VGT

Stmal --TA-LT RFGHDFPLTI-PV-EAL-EQVVL---LGEAKDS AMTVR--H--AH-GLSS'T

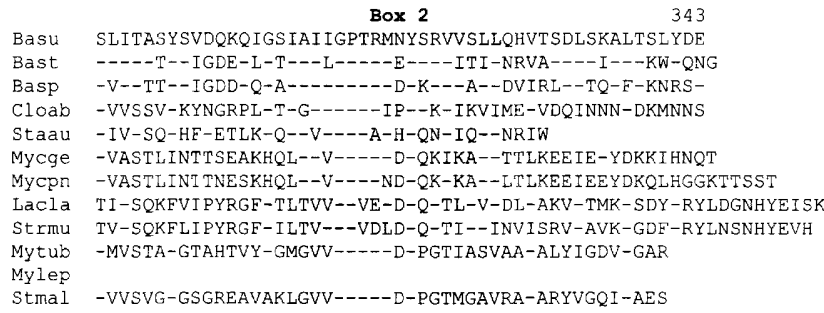

Fig. 3. Multiple alignment of hrcA-encoded sequences used in this study, from various Gram-positive bacteria. Abbreviations for species names are as follows: Basu, Bacillus subtilis; Bast, Bacillus stearothermophilus; Basp, Bacillus sphaericus; Cloac, Clostridium acetobutylicum; Staau, Staphylococcus aureus; Lacla, Lactococcus lactis; Mycge, Mycoplasma genitalium; Mycpn, Mycoplasma pneumoniae; Strmu, Streptococcus mutans; Mytub, Mycobacterium tuberculosis; Mylep, Mycobacterium leprae, and Stmal, Streptomyces albus. Amino acids
Lactococcus lactis and Streptococcus mutans cluster together and are not closely related to the Bacillus species. This is also shown by the organization of the dnaK operon, which contains the hrcA-grpE-dnaK genes but not the dnaJ gene (Fig. 2). Mycobacterium tuberculosis, Mycobacterium leprae and Streptomyces albus, all of which are Gram-positive bacteria with high $\mathrm{G}+\mathrm{C}$ DNA content, formed another cohesive group. The positioning of Synechocystis species on the basis of the $h r c A$ sequence suggests that the Grampositive bacteria do not form a monophyletic group. The positioning of Synechocystis species may be a consequence of the long branch effect (Felsenstein, 1985). Phylogenetic analyses carried out with all of the HrcA sequences resulted in a similar tree, except, of course, that Mycobacterium leprae was not represented (data not shown).

The tree exhibited that the three Bacillus species used in this study and Clostridium acetobutylicum share a common ancestor. This hierarchical arrangement demonstrates a close evolutionary relationship among these bacteria, which also share sporulation, a complex characteristic that involves the expression of several sporulation-dependent genes (Errington, 1993). The phylogenetic analyses derived from 16S rRNA gene sequences have not resolved the branching order within low-G +C DNA, Gram-positive bacteria (Olsen \& Woese, 1993; Stackebrandt et al., 1997; Woese, 1987). However, phylogenetic trees derived from amino acid sequence homologies of two proteins encoded by the dnaK operon (DnaK and DnaJ), as well as from Hsp60 sequences, place these organisms in separate clusters (Boorstein et al., 1994; Bustard \& Gupta, 1997; Gupta \& Golding, 1993) (see also Fig. 1).

The discrepancies in the branching orders noted above could be the result of several factors that may operate independently. It is known that some genes can tolerate fairly high mutation rates without adverse effects on their functioning. Consequently, mutation rates for the gene under study could vary dramatically in different organisms following their divergence. Thus, $d n a J$ and $h r c A$ homologues exhibit a minimum overall identity of $25 \%$, whereas the minimum identity exhibited by any two dnaK homologues is almost $45 \%$ (Bustard \& Gupta, 1997; Falah \& Gupta, 1994). It is also possible that the gene under study was acquired through horizontal gene transfer in one lineage at an earlier stage of divergence. It has been reported that some bacterial genera including Gram-positive bacteria contain more than one copy of dnaK and dnaJ homologues (Grandvalet et al., 1998; Nimura et al.,

are shown according to the standard one-letter code, and residues identical to those of $B$. subtilis are indicated by dashes. Gaps, indicated by blank spaces, are introduced in order to obtain maximum fit. The two highly conserved domains are labelled 'Box 1' and 'Box 2'. Asterisks highlight the insertions or deletions that could serve as 'signatures' in various sequences. 
Table 1. Similarity matrix of hrcA sequences

The pairwise amino acid identity $(\%)$ for the entire length of various $h r c A$ homologues was calculated by using the FASTA program and the PALIGN program from PC/GENE software.

\begin{tabular}{|lrrrrrrrrrrrr|}
\hline & $\mathbf{1}$ & $\mathbf{2}$ & $\mathbf{3}$ & $\mathbf{4}$ & $\mathbf{5}$ & $\mathbf{6}$ & $\mathbf{7}$ & $\mathbf{8}$ & $\mathbf{9}$ & $\mathbf{1 0}$ & $\mathbf{1 1}$ & $\mathbf{1 2}$ \\
\hline 1. Bacillus subtilis & 100 & & & & & & & & & & & \\
2. Bacillus stearothermophilus & 64 & 100 & & & & & & & & & & \\
3. Bacillus sphaericus & 56 & 56 & 100 & & & & & & & & & \\
4. Clostridium acetobutylicum & 35 & 35 & 33 & 100 & & & & & & & & \\
5. Staphylococcus aureus & 32 & 32 & 30 & 29 & 100 & & & & & & & \\
6. Mycoplasma genitalium & 31 & 29 & 30 & 27 & 27 & 100 & & & & & & \\
7. Mycoplasma pneumoniae & 32 & 29 & 29 & 29 & 26 & 80 & 100 & & & & \\
8. Lactococcus lactis & 27 & 28 & 26 & 26 & 25 & 26 & 26 & 100 & & & \\
9. Streptococcus mutans & 27 & 26 & 28 & 22 & 25 & 22 & 24 & 45 & 100 & & \\
10. Mycobacterium tuberculosis & 29 & 29 & 29 & 31 & 27 & 23 & 24 & 26 & 24 & 100 & & \\
11. Streptomyces albus & 27 & 28 & 27 & 30 & 28 & 25 & 27 & 24 & 24 & 56 & 100 & \\
12. Synechocystis species & 30 & 29 & 29 & 24 & 24 & 26 & 27 & 24 & 24 & 27 & 27 & 100 \\
\hline
\end{tabular}

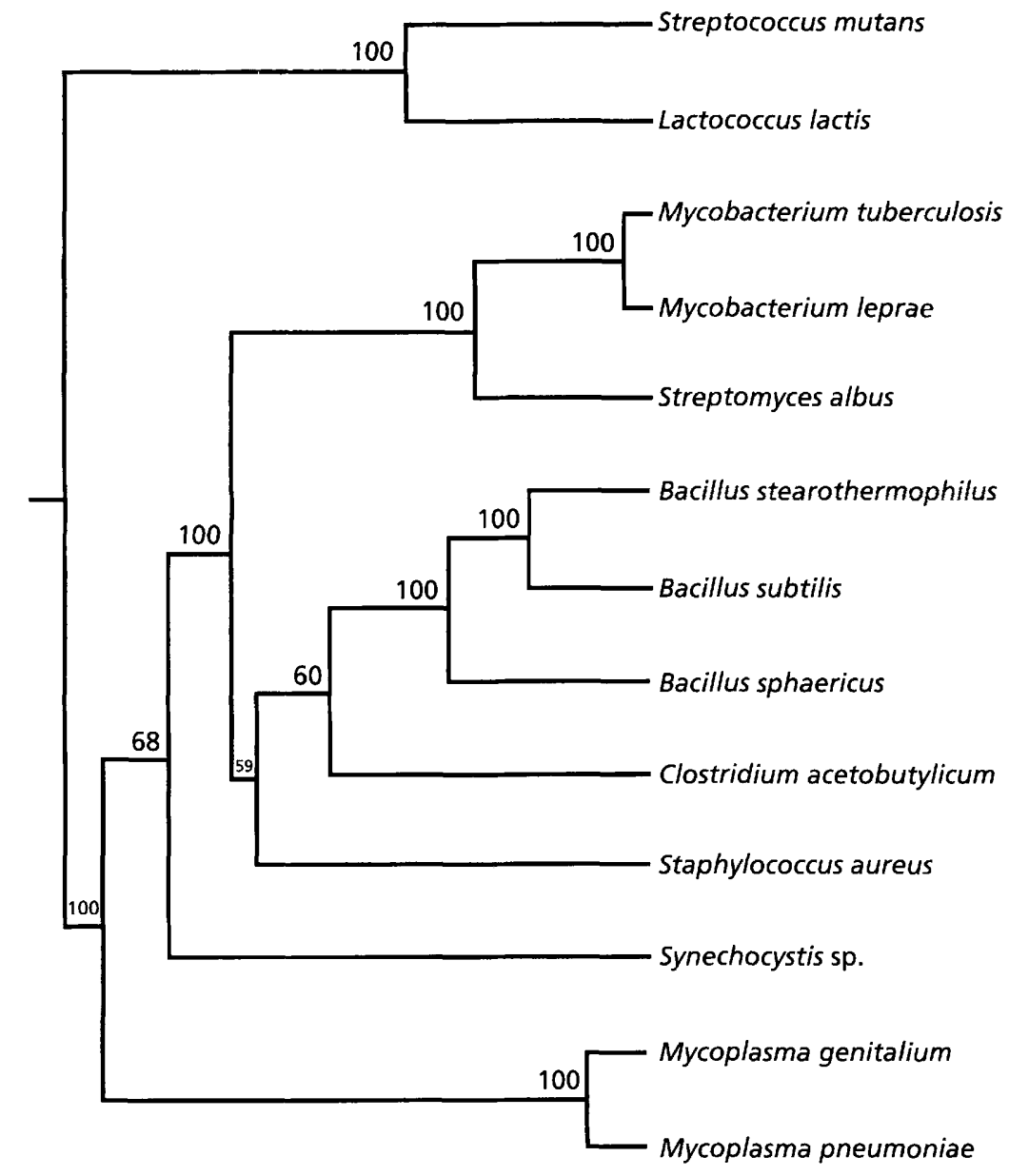

Fig. 4. Consensus, bootstrap, neighbourjoining, phylogenetic tree for Gram-positive bacteria based on HrcA sequences. The numbers on the nodes indicate the number of times (\%) the species (shown on the right) grouped together in 100 bootstrap samples. The phylogenetic analysis was carried out on the alignment of $195 \mathrm{~N}$ terminal amino acid residues of various HrcA sequences for which sequence information is available (including Mycobacterium leprae, as shown in Fig. 3).

1994; Ward-Rainey et al., 1997; Yoshimune et al., 1998). The extent of this duplication across various genera is not known at present. It is also unclear whether multiple copies originated in the same organism through gene duplication or were acquired by other mechanisms. It is probable that one of the gene copies was acquired through horizontal transfer in these genera. If so, this may explain some of the varying results obtained with $h r c A$, dnaJ and dnaK phylogenies. 
The overall homology of $\mathrm{HrcA}$ proteins roughly resembles that exhibited by DnaJ sequences (Bustard \& Gupta, 1997) and provides an alternative hierarchical order among Gram-positive bacteria. The availability of a greater number of phylogenetic trees based on a large number of conserved and ubiquitous cistrons is expected to allow the establishment of evolutionary relationships and hierarchical order among important bacterial groups, such as the Grampositive bacteria.

\section{REFERENCES}

Ahmad, S. \& Jensen, R. A. (1989). Utility of a bifunctional tryptophan pathway enzyme for the classification of the herbicola-agglomerans complex of bacteria. Int $J$ Syst Bacteriol 39, 100-104.

Altschul, S. F., Gish, W., Miller, W., Myers, E. W. \& Lipman, D. J. (1990). Basic local alignment search tool. J Mol Biol 215, 403-410.

Ballard, S. A., Go, M., Segers, R. P. A. M. \& Adler, B. (1998). Molecular analysis of the dna $\mathrm{K}$ locus of Leptospira interrogans serovar copenhageni. Gene 216, 21-29.

Boorstein, W. R., Ziegelhoffer, T. \& Craig, E. A. (1994). Molecular evolution of the HSP70 multigene family. J Mol Evol 38, 1-17.

Brown, J. R., Masuchi, Y., Robb, F. T. \& Doolittle, W. F. (1994). Evolutionary relationships of bacterial and archaeal glutamine synthetase genes. $J$ Mol Evol 38, 566-576.

Bucca, M., Ferina, G., Puglia, A. N. \& Smith, C. P. (1995). The dnaK operon of Streptomyces coelicolor encodes a novel heat-shock protein which binds to the promoter region of the operon. $\mathrm{Mol}$ Microbiol 17, 663-674.

Bustard, K. \& Gupta, R. S. (1997). The sequences of heat shock protein 40 (DnaJ) homologs provide evidence for a close evolutionary relationship between the Deinococcus-Thermus group and cyanobacteria. J Mol Evol 45, 193-205.

Cole, S. T., Brosch, R., Parkhill, J. \& 39 others (1998). Deciphering the biology of Mycobacterium tuberculosis from the complete genome sequence. Nature 393, 537-544.

Conway de Macario, E., Dugan, C. B. \& Macario, A. J. L. (1994). Identification of a grp $\mathrm{E}$ heat-shock gene homolog in the archaeon Methanosarcina mazei. J Mol Biol 240, 95-101.

Eaton, T., Shearman, C. \& Gasson, M. (1993). Cloning and sequence analysis of the dnaK gene region of Lactococcus lactis subsp. lactis. J Gen Microbiol 139, 3253-3264.

Errington, J. (1993). Sporulation in Bacillus subtilis: regulation of gene expression and control of morphogenesis. Microbiol Rev 57, 1-33.

Falah, M. \& Gupta, R. S. (1994). Cloning of the hsp 70/dnaK genes from Rhizobium meliloti and Pseudomonas cepacia: phylogenetic analyses of mitochondrial origin based on a highly conserved protein sequence. $J$ Bacteriol 176, 7748-7753.

Falah, M. \& Gupta, R. S. (1997). Phylogenetic analysis of mycoplasmas based on hsp70 sequences: cloning of the $d n a \mathrm{~K}$ (hsp70) gene region of Mycoplasma capricolum. Int J Syst Bacteriol 47, 38-45.

Felsenstein, J. (1985). Confidence limits in phylogenies: an approach using the bootstrap. Evolution 39, 783-791.
Fox, G. E., Wisotzkey, J. D. \& Jurtshuk, P., Jr (1992). How close is close: 16S rRNA sequence identity may not be sufficient to guarantee species identity. Int J Syst Bacteriol 42, 166-170.

Glatz, A., Horvath, I., Varvasovszki, V., Kovacz, E., Torok, Z. \& Vigh, L. (1997). Chaperonin genes of the Synechocystis PCC 6803 are differentially regulated under light-dark transition during heat stress. Biochem Biophys Res Commun 239, 291-297.

Grandvalet, C., Rapoport, G. \& Mazodier, P. (1998). $h r c A$, encoding the repressor of the groEL genes in Streptomyces albus $\mathrm{G}$, is associated with a second dnaJ gene. J Bacteriol $\mathbf{1 8 0}$, 5129-5134.

Gupta, R. S. (1995). Phylogenetic analysis of the $90 \mathrm{kd}$ heat shock family of protein sequences and an examination of the relationships between animals, plants and fungi species. $\mathrm{Mol}$ Biol Evol 12, 1063-1073.

Gupta, R. S. \& Golding, G. B. (1993). Evolution of HSP70 gene and its implications regarding relationships between archaebacteria, eubacteria and eukaryotes. $J$ Mol Evol 37, 573-582.

Gupta, R. S., Picketts, D. J. \& Ahmad, S. (1989). A novel ubiquitous protein 'chaperonin' supports the endosymbiotic origin of mitochondrion and plant chloroplast. Biochem Biophys Res Commun 163, 780-787.

Jayaraman, G. C., Penders, J. E. \& Burne, R. A. (1997). Transcriptional analysis of the Streptococcus mutans hrcA, grpE and dna $K$ genes and regulation of expression in response to heat shock and environmental acidification. Mol Microbiol 25, 329-341.

Jensen, R. A. \& Ahmad, S. (1990). Nested gene fusions as markers of phylogenetic branch points in prokaryotes. Trends Ecol Evol 5, 219-224.

Koch, B., Kilstrup, M., Vogensen, F. K. \& Hammer, K. (1998). Induced levels of heat shock proteins in a dnaK mutant of Lactococcus lactis. $J$ Bacteriol 180, 3873-3881.

Lipinska, B., King, J., Ang, D. \& Georgopoulos, C. (1988). Sequence analysis and transcriptional regulation of the Escherichia coli grp $\mathrm{E}$ gene, encoding a new heat shock protein. Nucleic Acids Res 16, 7545-7562.

Mogk, A. \& Schumann, W. (1997). Cloning and sequencing of the hrcA gene of Bacillus stearothermophilus. Gene 194, 133-136.

Myers, E. W. \& Miller, W. (1988). Optimal alignment in linear space. Comput Appl Biosci 4, 11-17.

Narberhaus, F., Giebeler, K. \& Bahl, H. (1992). Molecular characterization of the $d n a \mathrm{~K}$ gene region of Clostridium acetobutylicum, including grp $\mathrm{E}$, dnaJ and a new heat shock gene. J Bacteriol 174, 3290-3299.

Nimura, K., Yoshikawa, H. \& Takahashi, T. (1994). Identification of dnaK multigene family in Synechococcus sp. PCC 7942. Biochem Biophys Res Commun 201, 466-471.

Ohta, T., Saito, K., Kuroda, M., Honda, K., Hirata, H. \& Hayashi, H. (1994). Molecular cloning of two new heat shock genes related to the hsp70 genes in Staphylococcus aureus. J Bacteriol 176, 4779-4783.

Olsen, G. J. \& Woese, C. R. (1993). Ribosomal RNA: a key to phylogeny. FASEB J 7, 113-123.

Rainey, F. A., Burghardt, J., Kroppenstedt, R. M., Klatte, S. \& Stackebrandt, E. (1995). Phylogenetic analysis of the genera Rhodococcus and Nocardia and evidence for the evolutionary origin of the genus Nocardia from within the radiation of Rhodococcus species. Microbiology 141, 523-528.

Roberts, R. C., Toochinda, C., Avedissian, M., Baldini, R. L., Gomes, S. L. \& Shapiro, L. (1996). Identification of a Caulobacter 
crescentus operon encoding $h r c A$, involved in negatively regulating heat-inducible transcription and the chaperone gene grpE. J Bacteriol 178, 1829-1841.

Saito, H. \& Uchida, H. (1978). Organization and expression of the dnaJ and dnaK genes of Escherichia coli K12. Mol Gen Genet 164, 1-8.

Schulz, A. \& Schumann, W. (1996). hrcA, the first gene of the Bacillus subtilis dnaK operon encodes a negative regulator of class I heat shock genes. J Bacteriol 178, 1088-1093.

Stackebrandt, E. \& Goebel, B. M. (1994). A place for DNA-DNA reassociation and $16 \mathrm{~S}$ rRNA sequence analysis in the present species definition in bacteriology. Int $J$ Syst Bacteriol 44, 846-849.

Stackebrandt, E., Rainey, F. A. \& Ward-Rainey, N. L. (1997). Proposal for a new hierarchic classification system, Actinobacteria classis nov. Int $J$ Syst Bacteriol 47, 479-491.

Tan, M., Wong, B. \& Engel, J. N. (1996). Transcriptional organization and regulation of the $d n a \mathrm{~K}$ and gro $\mathrm{E}$ operons of Chlamydia trachomatis. J Bacteriol 178, 6983-6990.
Tokunaga, H., Yamakawa, M., Mizukami, M., Takagi, H. \& Tokunaga, M. (1998). Molecular cloning of the dnaK locus, and purification and characterization of a DnaK protein from Bacillus brevis HPD31. Biochim Biophys Acta 1387, 65-79.

Ward-Rainey, N., Rainey, F. A. \& Stackebrandt, E. (1997). The presence of a dnaK (HSP70) multigene family in members of the orders Planctomycetales and Verrucomicrobiales. J Bacteriol 179, 6360-6366.

Wetzstein, M., Volker, U., Dedio, J., Loban, S., Zuber, U., Schiesswohl, M., Herget, C., Hecker, M. \& Schumann, W. (1992). Cloning, sequencing and molecular analysis of the dna $\mathrm{K}$ locus from Bacillus subtilis. J Bacteriol 174, 3300-3310.

Woese, C. R. (1987). Bacterial evolution. Microbiol Rev 51, 221-271.

Woese, C. R. (1994). There must be a prokaryote somewhere: microbiology's search for itself. Microbiol Rev 58, 1-9.

Yoshimune, K., Yoshimura, T. \& Esaki, N. (1998). Hsc62, a novel DnaK homologue of Escherichia coli. Biochem Biophys Res Commun 250, 115-118. 